\title{
Prognostic Factors Of Patients With Unexpected Pleural Dissemination During Thoracoscopic Surgery For Non-Small Cell Lung Cancer: A Retrospective Study
}

Jin-xiao Liang ( $\square$ liangjinxiaozch@163.com )

Cancer Hospital of the University of Chinese Academy of Sciences (Zhejiang Cancer Hospital)

Wei-tian Wei

Cancer Hospital of the University of Chinese Academy of Sciences (Zhejiang Cancer Hospital)

Wei Gao

Zhejiang University City College

Xun Yang

Cancer Hospital of the University of Chinese Academy of Sciences (Zhejiang Cancer Hospital) Jin-shi Liu

Cancer Hospital of the University of Chinese Academy of Sciences (Zhejiang Cancer Hospital)

\section{Research Article}

Keywords: prognostic factors, pleural dissemination, non-small cell lung cancer, surgery

Posted Date: March 9th, 2022

DOI: https://doi.org/10.21203/rs.3.rs-805667/v2

License: (c) (i) This work is licensed under a Creative Commons Attribution 4.0 International License.

Read Full License 


\section{Abstract}

Background: Non-small cell lung cancer (NSCLC) is one of the most common malignant tumors with high degree of malignancy and early metastasis. A preoperative examination is undetectable when pleural dissemination of NSCLC occurs at an early stage, leading surgeons to detect pleural dissemination during surgery. However, there are few studies on the prognostic factors of NSCLC patients with pleural disseminated during surgery.

Methods: We retrospectively analyzed 54 patients with NSCLC with pleural dissemination found during video-assisted thoracoscopic surgery to investigate the effects of clinical-pathological features, serum characteristics, surgical methods, and postoperative treatment on their prognosis.

Results and conclusion: We found that squamous cell carcinoma $(p=0.008)$, high level of serum GGT $(p=0.046)$ and CA199 ( $p=0.001)$ were significantly correlated with the poor prognosis of NSCLC with pleural dissemination. Resection of the primary tumor was not necessary for patients who could receive targeted therapy after surgery, while for patients who receive chemotherapy after surgery, resection of the primary tumor, especially lobectomy, could obtain a better prognosis. Targeted therapy is preferred if there is a driving gene mutation after the operation, and immunotherapy combined with chemotherapy can be selected if there is no mutation. These results can provide a clinical basis for prognosis judgment and treatment decision of NSCLC patients with pleural dissemination found during the operation.

\section{Background}

Lung cancer is a highly malignant cancer that threatens human lives and has the highest cancer mortality rate among all cancer species ${ }^{[1]}$. Non-small cell lung cancer (NSCLC) is the most common type of lung cancer, representing roughly $85 \%$ of all lung cancer cases ${ }^{[2]}$. Because of its insidious onset, lymph node metastasis and hematogenous metastasis can occur at an early stage, which leads to an unsatisfactory prognosis of NSCLC. At present, surgical treatment is still the main treatment for earlystage and locally advanced resectable NSCLC ${ }^{[3]}$. However, it is still a controversial issue whether to resect the primary lesion in patients with NSCLC who accidentally discover pleural dissemination during surgery ${ }^{[4,5]}$.

In the 8th edition of the Union for International Cancer Control (UICC)/ the American Joint Committee on Cancer (AJCC) on lung cancer's TNM staging system, pleural dissemination was classified as M1 $a^{[6]}$. The prognosis of patients with M1a stage NSCLC is poor, the median survival time is only 11.5 months, and surgery is usually not recommended to treat patients in this stage ${ }^{[7]}$. However, in some NSCLC patients, pre-operative examinations are often undetectable in the early stages of pleural dissemination, leading to surgeons accidentally detecting it during surgery. Studies showed that primary tumor resection could prolong the prognosis of ipsilateral pleural disseminated NSCLC patients who can not be targeted for therapy ${ }^{[8,9]}$. Li et al. found that PFS and OS improved after primary lesion and visible pleural nodule resection in patients with lung adenocarcinoma with unexpected pleural dissemination 
intraoperatively ${ }^{[10]}$. These studies showed that primary tumor resection could prolong the survival time of NSCLC patients, but because of the limitation of sample size, more related studies are needed for further confirmation. The present study explored the prognostic factors of NSCLC patients with unexpected pleural dissemination during thoracoscopic surgery, providing the basis for the treatment and prognosis prediction of these patients.

\section{Methods}

\section{Patients}

All patients with lung cancer underwent video-assisted thoracoscopic surgery, and pleural dissemination was accidentally detected during the surgery in the Cancer Hospital of the University of Chinese Academy of Sciences (Zhejiang Cancer Hospital) from Jan 2009 to Apr 2019. Pleural dissemination was confirmed pathologically in all cases. Cases with pathological results of small cell lung cancer were excluded. The current disease progression and survival state of the patients were confirmed through outpatient medical records, examination results, and telephone follow-up. All procedures for data extraction and follow-up were ratified by the Ethics Committee of Zhejiang Cancer Hospital.

\section{Data Extraction And Synthesis}

Two authors performed the case data extraction independently. The following data were extracted from each electronic medical record: age, gender, history of hypertension, history of diabetes, body mass index (BMI), family history of lung cancer, operative methods, tumor location, tumor size, tumor pathological types, alkaline phosphatase (ALP), L- $\gamma$-glutamyltransferase (GGT), lactate dehydrogenase (LDH), creatine kinase (CK), cytokeratin 19 (CK-19), carcinoembryonic antigen (CEA), carbohydrate antigen 125 (CA125) and carbohydrate antigen 199 (CA199). Clinical T stage (cT), clinical N stage (cN) and clinical stage (cTNM) were extracted from CT images. The cancers were staged according to the 8th edition of the UICC/ AJCC on lung cancer's TNM staging system ${ }^{[6]}$.

In data analysis, all factors were conducted as dichotomous variables, as $<60$ years old versus $\geq 60$ years old for age; male versus female for gender; $<21$ versus $\geq 21$ for $\mathrm{BMl}$; with versus without for history of hypertension, history of diabetes and family history of lung cancer; peripheral versus central for tumor location; $<3 \mathrm{~cm}$ versus $\geq 3 \mathrm{~cm}$ for tumor size; adenocarcinoma versus squamous cell carcinoma for tumor pathological types; cT1 versus cT2 4 for cT stage; $\mathrm{CNO}$ versus $\mathrm{CN} 1 \sim 2$ for $\mathrm{cN}$ stage; I stage versus II III stage for cTNM stage; $<125 \mathrm{U} / \mathrm{L}$ versus $\geq 125 \mathrm{U} / \mathrm{L}$ for $A L P ;<60 \mathrm{U} / \mathrm{L}$ versus $\geq 60 \mathrm{U} / \mathrm{L}$ for $\mathrm{GGT}$; $<240 \mathrm{U} / \mathrm{L}$ versus $\geq 240 \mathrm{U} / \mathrm{L}$ for $\mathrm{LDH} ;<190 \mathrm{U} / \mathrm{L}$ versus $\geq 190 \mathrm{U} / \mathrm{L}$ for $\mathrm{CK}_{;}<3.3 \mathrm{ng} / \mathrm{ml}$ versus $\geq 3.3 \mathrm{ng} / \mathrm{ml}$ for CK- $19 ;<5 \mathrm{ng} / \mathrm{ml}$ versus $\geq 5 \mathrm{ng} / \mathrm{ml}$ for CEA; $<35 \mathrm{U} / \mathrm{ml}$ versus $\geq 35 \mathrm{U} / \mathrm{ml}$ for CA $125 ;<37 \mathrm{U} / \mathrm{ml}$ versus $\geq$ $37 \mathrm{U} / \mathrm{ml}$ for CA199. Two factors were conducted as ternary variables, as open-close surgery, lobectomy surgery versus pulmonary wedge resection for operative methods; without treatment, targeted therapy versus chemotherapy for postoperative treatment. 


\section{Statistical Analysis}

SPSS 23.0 statistical software was used for statistical analysis. The overall survival curves were produced using the Kaplan-Meier method, the difference in survival was determined using the log-rank test. Differences were considered to indicate a statistically significant result with a P-value $<0.05$.

\section{Results}

\section{Clinicopathological features of NSCLC patients}

Through case review, 54 patients with NSCLC were incidentally found to have pleural dissemination during video-assisted thoracoscopic surgery (Table.1). The mean age for these patients was 58.7 years $( \pm 9.4)$ that range of age was 31-74 years. 32 of them were male, other 22 patients were female. About past history, 19 patients had hypertension, 4 patients had diabetes and 7 patients had family history of lung cancer. The mean BMI was $23.5( \pm 3.1)$ that range of 18.4 to 30.5 . In tumor location, 8 patients were central NSCLC and 46 were peripheral. 7 of NSCLC were squamous cell carcinoma and other 47 were adenocarcinoma. In tumor size, 30 NSCLC were less than $3 \mathrm{~cm}$ and other 24 were greater than $3 \mathrm{~cm}$. In cT stage, 19 were T1, 31 were T2, 3 were T3 and 1 was T4. In cN stage, 44 were N0, 7 were N1 and 3 were N2. In cTNM stage, 16 were I stage, 33 were II stage and 5were III stage.

\section{Serum Features Of Nsclc Patients}

In serum features analysis (Table.2), the range of ALP was $50-151 \mathrm{U} / \mathrm{L}$ and the median was $82.4 \mathrm{U} / \mathrm{L}$. The range of GTT was $6-212 \mathrm{U} / \mathrm{L}$ and the median was $36.7 \mathrm{U} / \mathrm{L}$. LDH was $120-292 \mathrm{U} / \mathrm{L}$ and the median was 191.2U/L. CK was 28-659U/L and the median was $102.6 \mathrm{U} / \mathrm{L}$. CK-19 was $0.6-10 \mathrm{ng} / \mathrm{ml}$ and the median was $3.4 \mathrm{ng} / \mathrm{ml}$. CEA was $0.7-3212.7 \mathrm{ng} / \mathrm{ml}$ and the median was $74.6 \mathrm{ng} / \mathrm{ml}$. CA- 125 was $5.7-414.8 \mathrm{U} / \mathrm{ml}$ and the median was $42.5 \mathrm{U} / \mathrm{ml}$. CA-199 was $2-248.2 \mathrm{U} / \mathrm{ml}$ and the median was $23.9 \mathrm{U} / \mathrm{ml}$.

\section{Treatment Methods Of Nsclc Patients}

In video-assisted thoracoscopic surgery, 19 patients underwent open-close surgery, 8 underwent lobectomy, and other 27 underwent pulmonary wedge resection. In postoperative treatment, 5 patients did not receive treatment, 35 patients received targeted therapy alone or combined therapy including targeted therapy, and 14 patients received chemotherapy alone (Table 3). 
Table 1

Clinicopathological features of NSCLC patients

\begin{tabular}{|ll|}
\hline Variables & Number(\%) \\
\hline Total & $54(100 \%)$ \\
\hline Age, y (mean \pm SD) & $58.7 \pm 9.4$ \\
\hline BMI (mean \pm SD) & $25.3 \pm 3.1$ \\
\hline Gender & $32(59.3)$ \\
\hline Male & $22(40.7)$ \\
\hline Female & \\
\hline Hypertension history & $19(35.2)$ \\
\hline With & $35(64.8)$ \\
\hline Without & \\
\hline Diabetes history & $4(7.4)$ \\
\hline With & $50(92.6)$ \\
\hline Without & \\
\hline NSCLC family history & $7(13.0)$ \\
\hline With & $47(87.0)$ \\
\hline Without & \\
\hline Tumor location & \\
\hline Center & \\
\hline Peripheral & \\
\hline Tumor pathological types & \\
\hline Squamous cell carcinoma & $74.8)$ \\
\hline Adenocarcinoma & \\
\hline Tumor size & \\
\hline S3cm & \\
\hline cT stagem & \\
\hline
\end{tabular}




\begin{tabular}{|ll|}
\hline Variables & Number(\%) \\
\hline cT2 & $31(57.3)$ \\
\hline CT3 & $3(5.6)$ \\
\hline CT4 & $1(1.9)$ \\
\hline CN stage & \\
CN0 & $44(81.4)$ \\
CN1 & $7(13.0)$ \\
CN2 & $3(5.6)$ \\
CTNM stage & \\
\hline I stage & $16(29.6)$ \\
\hline II stage & $33(61.1)$ \\
\hline III stage & $5(9.3)$ \\
\hline SD: standard deviation & \\
\hline
\end{tabular}


Table 2

Serum features of NSCLC patients

\begin{tabular}{|ll|}
\hline Variables & Number(\%) \\
\hline Total & $54(100)$ \\
\hline ALP U/L (mean, range) & $82.4,50-151$ \\
\hline$<125$ & $51(94.4)$ \\
\hline$\geq 125$ & $3(5.6)$ \\
\hline GGT U/L (mean, range) & $36.7,6-212$ \\
\hline$<60$ & $46(85.2)$ \\
\hline$\geq 60$ & $8(14.8)$ \\
\hline LDH U/L (mean, range) & $191.2,120-292$ \\
\hline$<240$ & $47(87.0)$ \\
\hline$\geq 240$ & $7(13.0)$ \\
\hline CK U/L (mean, range) & $102.6,28-659$ \\
\hline$<190$ & $52(96.3)$ \\
\hline$\geq 190$ & $2(3.7)$ \\
\hline CK-19 ng/ml (mean, range) & $3.4,0.6-10$ \\
\hline$<3.3$ & $35(64.8)$ \\
\hline$\geq 3.3$ & $19(35.2)$ \\
\hline CEA ng/ml (mean, range) & 74.6, \\
\hline$<5$ & $0.7-3212.7$ \\
\hline$\geq 5$ & $30(55.6)$ \\
\hline CA125 U/ml (mean, range) & $24(44.4)$ \\
\hline$<35$ & $42.5,5.7-414.8$ \\
\hline$\geq 35$ & $40(74.1)$ \\
\hline CA199 U/ml (mean, range) & $23.9,2-248.2$ \\
\hline$<37$ & $48(25.9)$ \\
\hline & $67.9)$ \\
\hline
\end{tabular}


Table 3

Treatment methods of NSCLC patients

\begin{tabular}{|lll|}
\hline Variables & Number (\%) & 2-years survival (\%) \\
\hline Total & $54(100)$ & $25 / 54(46.3)$ \\
\hline Surgical methods & & \\
\hline Open-close surgery & $19(35.2)$ & $8 / 19(42.1)$ \\
\hline Pulmonary wedge resection & $27(50.0)$ & $11 / 27(40.7)$ \\
\hline Lobectomy & $8(14.8)$ & $6 / 8(75)$ \\
\hline Postoperative treatment & & \\
\hline Without treatment & $5(9.3)$ & $0 / 5(0.0)$ \\
\hline Targeted therapy & $35(64.8)$ & $22 / 35(62.9)$ \\
\hline Chemotherapy & $14(25.9)$ & $3 / 14(21.4)$ \\
\hline
\end{tabular}

\section{Survival Analysis Of Each Factors}

Since the most recent case was operated on in March 2019, we used 2-year survival as the follow-up endpoint. In survival analysis, we found that age $(p=0.335)($ Fig. 1A), gender $(p=0.399)$ (Fig. 1B), diabetes history $(p=0.356)$ (Fig. 1D), BMI $(p=0.651)$ (Fig. 1E), family history $(p=0.758)$ (Fig. 1F), tumor size $(p=0.383)($ Fig. 1G), tumor location $(p=0.380)($ Fig. 1H), T stage $(p=0.941)($ Fig. 1I), ALP $(p=0.958)$ (Fig. 2A), LDH ( $p=0.724)$ (Fig. $2 C), C K(p=0.836)$ (Fig. 2D), CK-19 $(p=0.654)$ (Fig. 2E), CEA $(p=0.662)$ (Fig. 2F), were not associated with prognosis. There were no significant difference between hypertension history $(p=0.128)($ Fig. $1 C)$, N stage $(p=0.270)$ (Fig. 1J), TNM stage $(p=0.160)$ (Fig. 1K), CA125 $(p=$ 0.092 ) (Fig. $2 G)$, surgical methods $(p=0.289)$ (Fig. $3 A$ ), and prognosis of NSCLC patients. However, tumor pathological types $(p=0.008)$ (Fig. 1L), GTT $(p=0.046)($ Fig. 2B), CA199 $(p=0.001)(F i g .2 H)$, postoperative treatment $(p=0.000)$ (Fig. 3B), were obviously correlated with prognosis of NSCLC patients with unexpected pleural dissemination during thoracoscopic surgery.

\section{Discussion}

In some NSCLCs, the pleura can be invaded at an early stage, causing cancer cells to break through the pleura and spread to the visceral pleura or parietal pleura, leading to the advanced stage of the primary tumor at a relatively small size. Pleural dissemination was classified as stage IV according to the UICC/AJCC lung cancer stage and had no surgical indication ${ }^{[6]}$. Computed tomography (CT) can detect most pleural involvement and malignant pleural effusion ${ }^{[11]}$. However, in the early stage of pleural 
dissemination, CT may appear false negative, which leads to the surgeon accidentally discovering pleural dissemination during surgery ${ }^{[10]}$.

Lida et al. reported that the median survival time and 5-year survival rate of NSCLC with pleural carcinomatosis and without other metastatic disease were 34 months and $29.3 \%{ }^{[12]}$. Chiang's study reported that the 5-year survival rate and median survival time were $30.2 \%$ and 29.3 months $^{[13]}$. In this study, the 2-year survival rate was $46.3 \%$ (25/54). Although the survival time of NSCLC patients with pleural disseminated which was found during surgery was significantly longer than other stage IV patients $^{[14]}$, the median survival time was merely about 30 months. It is important to find the prognostic factors for predicting the prognosis of patients, but there are no relevant reports yet. In this study, we found that groups with hypertension history, cN1 2, cll III stage, high level of CA125 had a worse prognosis than groups without hypertension history, cN0, cl stage, low level of CA125, but the difference was not significant. Cardiovascular disease is the leading cause of late morbidity and mortality among cancer survivors, and hypertension is associated with the survival of tumor patients ${ }^{[15]}$. Zeng et al. reported that complications of hypertension might confer a poor survival for advanced NSCLC patients ${ }^{[16]}$. In our study, we found that the survival time of patients with hypertension was shorter than that of patients without hypertension, which was also similar to the results of other related reports. $\mathrm{CN}$ and CTNM stages are closely related to the prognosis of patients with NSCLC ${ }^{[6]}$. However, in this study, we enrolled all patients with M1 stage and their pathological stages were stage IV, which resulted in no significant difference between $\mathrm{CN}$ and cTNM stages and the prognosis of lung cancer. Our results showed that the survival time of patients with increased expression of tumor-associated antigen CA-125 was shortened, but the difference was not significant in this study. Unfortunately, age, gender, diabetes history, BMI, family history, tumor size, tumor location, CT stage, ALP, LDH, CK, CK-19 and CEA were not associated with lung cancer survival. However, we found that tumor pathological types and serum levels of GGT and CA199 were significantly correlated with the prognosis of NSCLC with pleural dissemination. The 2-year survival rate of adenocarcinoma $(51.1 \%, 24 / 47)$ was significantly higher than that of squamous cell carcinoma $(14.3 \%, 1 / 7)$, which might be due to the fact that squamous cell carcinoma had few gene mutations and could only be treated with chemotherapy after the operation, which was less effective than targeted therapy after operation for most adenocarcinomas. Although GTT is an indicator of liver function, it has been proved to be related to oxidative stress ${ }^{[17]}$, which may also be related to the occurrence and development of tumors. We were also surprised to find that the survival time of patients with abnormal GTT increase is shorter than that of the normal group, which indicates that GTT was related to the prognosis of NSCLC patients. The tumor marker CA199 is a sensitive marker for pancreatic, gastric and hepatobiliary malignancies. However, it had less report on the correlation with the prognosis of NSCLC. We confirmed that the prognosis of patients with increased serum CA199 levels decreased significantly, which indicated that CA199 might become a prognostic predictor of patients with pleural disseminated NSCLC found during the operation.

Whether a surgeon should perform resection of a primary tumor when the pleural spread is accidentally detected during surgery has also become a hot topic of research. Some doctors believed that NSCLC with 
pleural dissemination was stage IV, and there was no indication for surgery. The primary tumor could be used as the evaluation standard of postoperative treatment effect. Therefore, surgical resection of the primary tumor was not recommended. Other doctors believed that primary tumor resection during operation reduced tumor burden and prolonged survival time of NSCLC patients. Positive results have been obtained in several studies that suggest prolonged survival after resection of the primary tumor ${ }^{[8-}$ 10]. However, in this study, we found that two-year overall survival after lobectomy $(75 \%, 6 / 8)$ was significantly higher than patients with pulmonary wedge resection $(40.7 \%, 11 / 27)$ and open-close surgery $(42.1 \%, 8 / 19)$, but there was no difference between pulmonary wedge resection and without tumor resection. Our result differs from the previous positive results ${ }^{[8-10]}$ in that there was no clear advantage of pulmonary wedge resection for NSCLC with pleural dissemination found during the operation. In further analysis, we found that different surgical methods in NSCLC patients receiving targeted therapy had similar 2-year survival rates (lobectomy was $66.7 \%$, pulmonary wedge resection was $62.5 \%$ and openclose surgery was $61.5 \%)$. However, the 2-year survival rate of patients receiving chemotherapy after lobectomy $(100 \%, 2 / 2)$, pulmonary wedge resection $(12.5 \%, 1 / 8)$ and open-close surgery $(0,0 / 4)$ were different. These results suggested that primary tumor resection did not have a significant impact on the prognosis of postoperative targeted therapy NSCLC with pleural dissemination patients. However, it improved the prognosis of patients undergoing chemotherapy after surgery, and the prognosis of lobectomy was better than that of pulmonary wedge resection. Due to the limited number of cases we included, additional cases need to be collected for further justification.

Chemotherapy, targeted therapy and immunotherapy are the common treatment methods for M1 stage NSCLC [3]. Since our cases were collected 2 years ago, when immunotherapy was just emerging and costly in China, no patient we included underwent immunotherapy. We performed gene testing on patients with adenocarcinoma. Targeted therapy was preferred if a driver gene mutation was detected. Chemotherapy was given if there was no mutation in adenocarcinoma patients and all squamous cell carcinoma patients. However, some patients with adenocarcinoma did not undergo genetic testing for economic reasons and directly received chemotherapy or abandoned treatment. Our results showed that the 2-year survival rate without treatment was $0(0 / 5), 21.4 \%(3 / 14)$ with chemotherapy and $62.9 \%$ $(22 / 35)$ with targeted therapy. It indicated that the survival time of NSCLC patients with pleural dissemination receiving targeted therapy was significantly longer than that of patients receiving chemotherapy, and targeted therapy might be the first choice if the tumor had the driving gene mutations. However, if there were no driver gene mutations, immunotherapy combined with chemotherapy could be tried and better results could be obtained.

\section{Conclusions}

In summary, we found that squamous cell carcinoma, high level of serum GGT and CA199 were significantly correlated with the poor prognosis of NSCLC with pleural dissemination. When pleural dissemination is found during surgery in patients with NSCLC, the primary tumor resection is not required if the tumor has a driver gene mutation. However, if there is no driver gene mutation, the primary tumor 
resection, especially lobectomy, is recommended. It also requires surgeons to puncture the tumor preoperatively to identify the pathology and perform genetic testing. Targeted therapy is preferred if there is a driving gene mutation after the operation, and immunotherapy combined with chemotherapy can be selected if there is no mutation. This study mainly analyzes the prognostic factors, surgical methods and postoperative treatment of NSCLC with pleural dissemination found during the operation, and provides the clinical basis for the diagnosis and treatment of this kind of NSCLC in the future. However, due to the limitated sample size of this study, it is necessary to collect further samples for analysis and draw more reliable conclusions.

\section{Abbreviations}

NSCLC: non-small cell lung cancer; UICC: Union for International Cancer Control; AJCC: American Joint Committee on Cancer; BMI: body mass index; ALP: alkaline phosphatase; GGT: L-y-glutamyltransferase; LDH: lactate dehydrogenase; CK: creatine kinase; CK-19: cytokeratin 19; CEA: carcinoembryonic antigen; CA-125: carbohydrate antigen 125; CA-199: carbohydrate antigen 199.

\section{Declarations}

\section{Ethics approval and consent to participate}

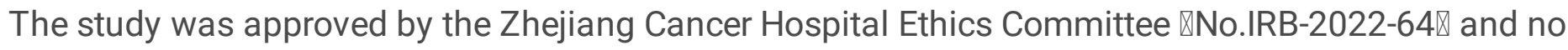
additional administrative permissions were required to access the raw data. Given the retrospective design, a waiver of participant consent was granted by the ethics committee.

\section{Consent for publication}

Not applicable

\section{Availability of data and materials}

The datasets supporting the conclusion of this article are included within the article.

\section{Competing interests}

The authors declare no conflict of interest.

\section{Funding}

This work was supported by grants from Zhejiang Province Public Welfare Technology Application Research Project (Animal Experiment Project) (No. LGD20H160002) and Medical Health Science and Technology Project of Zhejiang Provincial Health Commission (Nos. 2020KY083, 2020KY680, 2018KY022, 2017KY237).

\section{Authors' contributions}


Jin-xiao Liang wrote the manuscript. Wei-tian Wei and Wei Gao extracted and synthesized the data. Xun Yang processed the data analysis. Jin-shi Liu revised the final manuscript.

\section{Acknowledgements}

This work was supported by grants from Zhejiang Province Public Welfare Technology Application Research Project (Animal Experiment Project) (No. LGD20H160002) and Medical Health Science and Technology Project of Zhejiang Provincial Health Commission (Nos. 2020KY083, 2020KY680, 2018KY022, 2017KY237).

\section{References}

1. Siegel RL, Miller KD, Jemal A. Cancer statistics, 2020. CA Cancer J Clin. 2020; 70(1): 7-30.

2. Gridelli C, Rossi A, Carbone DP, et al. Non-small-cell lung cancer. Nat Rev Dis Primers. 2015; 1:15009.

3. Duma N, Santana-Davila R, Molina JR. Non-small cell lung cancer: epidemiology, screening, diagnosis, and treatment. Mayo Clin Proc. 2019; 94(8): 1623-1640.

4. Chambers $A$, Routledge T, Billè $A$, Scarci M. Does surgery have a role in T4N0 and T4N1 lung cancer? Interact Cardiovasc Thorac Surg. 2010; 11(4): 473-479.

5. Alfonso Fiorelli, Mario Santini. In lung cancer patients where a malignant pleural effusion is found at operation could resection ever still be justified? Interact Cardiovasc Thorac Surg. 2013; 17(2): 407412.

6. Nicholson AG, Tsao MS, Travis WD, et al. Eighth Edition Staging of Thoracic Malignancies: Implications for the Reporting Pathologist. Arch Pathol Lab Med. 2018; 142(5): 645-661.

7. Eberhardt WE, Mitchell A, Crowley J, et al. The IASLC Lung Cancer Staging Project: Proposals for the Revision of the M Descriptors in the Forthcoming Eighth Edition of the TNM Classification of Lung Cancer. J Thorac Oncol. 2015; 10(11): 1515-1522.

8. Hu J, Chen Y, Zhu X, et al. Surgical choice of non-small cell lung cancer with unexpected pleural dissemination intraoperatively. BMC Cancer. 2021; 21(1): 445.

9. Li H, Liu T, Sun Z, Yang F. Primary tumor resection of non-small cell lung cancer patients with ipsilateral pleural dissemination (M1a) in the era of targeted therapy. Thorac Cancer. 2020; 11(11): 3213-3222.

10. Li C, Kuo SW, Hsu HH, Lin MW, Chen JS. Lung adenocarcinoma with intraoperatively diagnosed pleural seeding: Is main tumor resection beneficial for prognosis? J Thorac Cardiovasc Surg. 2018; 155(3): 1238-1249.

11. Zhu P, Bai H, Yi F, Miao J, Yu J, Yang Z. CT features and anatomic distribution of pleural dissemination in lung cancer. Zhongguo Fei Ai Za Zhi. 2003; 6(1): 26-29.

12. lida T, Shiba M, Yoshino I, et al. Surgical intervention for non-small-cell lung cancer patients with pleural carcinomatosis: results from the Japanese lung cancer registry in 2004. J Thorac Oncol. 2015; 10(7): 1076-1082. 
13. Chiang $\mathrm{CL}$, Wang LC, Ho HL, et al. Effect of postoperative systemic therapy on pulmonary adenocarcinoma with unexpected pleural spread detected during thoracotomy or thoracoscopy. Oncotarget. 2017; 9(4): 5435-5444.

14. Takano N, Ariyasu R, Koyama J, et al. Improvement in the survival of patients with stage IV nonsmall-cell lung cancer: experience in a single institutional 1995-2017. Lung Cancer. 2019; 131: 6977.

15. Satoshi Kidoguchi, Naoki Sugano, Gorou Tokudome, et al. New concept of onco-hypertension and future perspectives. Hypertension. 2021; 77(1): 16-27.

16. Zeng $X$, Zeng $D$, Cheng J, et al. Influence of hypertension on the survival of non-small cell lung cancer patients with type 2 diabetes mellitus. Med Sci Monit. 2020; 26: e921676.

17. Takigawa T, Hibino Y, Kimura S, et al. Association between serum gamma-glutamyltransferase and oxidative stress related factors. Hepatogastroenterology. 2008; 55(81): 50-53.

\section{Figures}
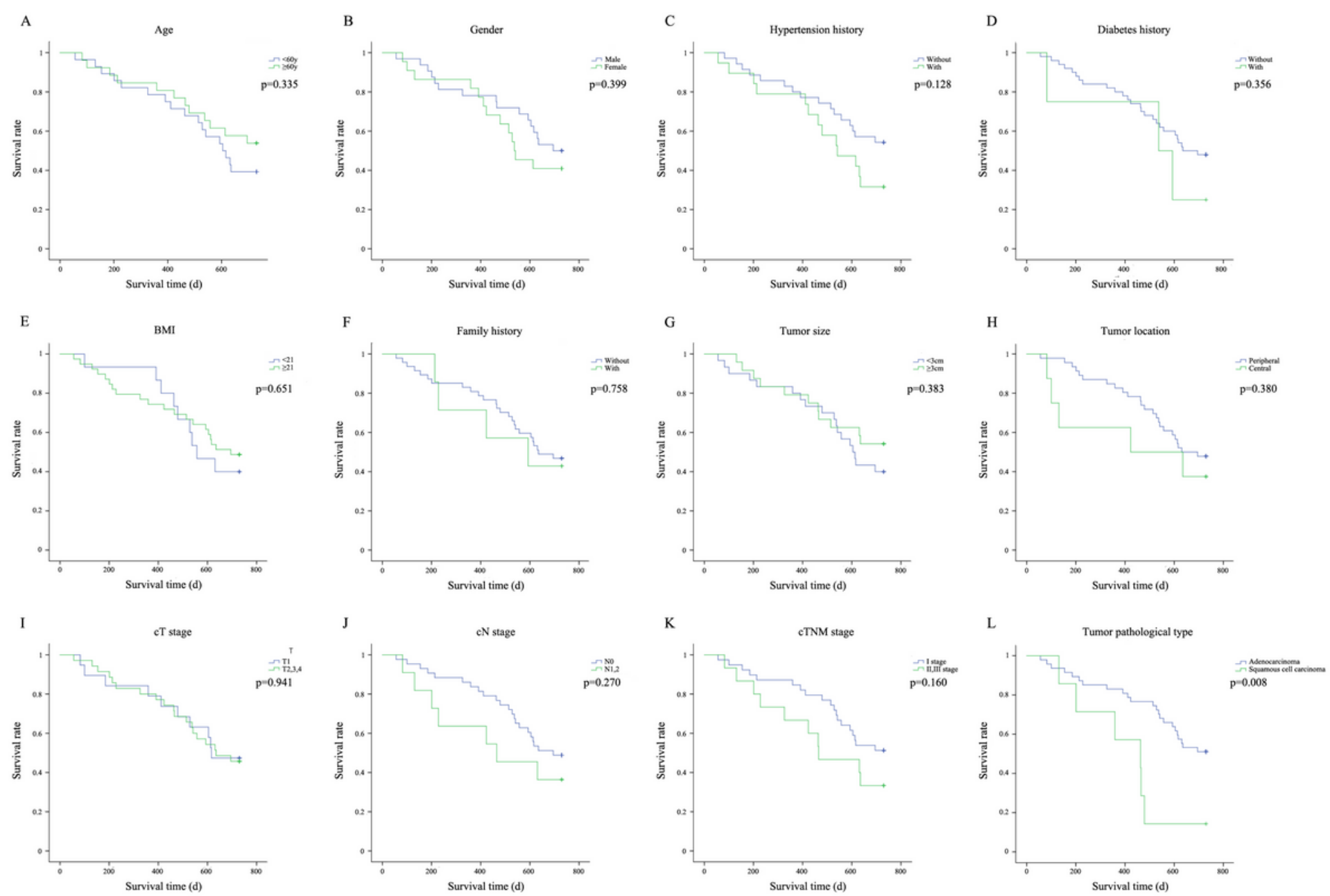

\section{Figure 1}


Kaplan-Meier survival curve of clinical-pathological factors for 54 NSCLC patients with unexpected pleural dissemination during thoracoscopic surgery. (A) age: <60y (blue) or $\geq 60 y$ (green); (B) gender: male (blue) or female (green); (C) hypertension history: without (blue) or with (green); (D) diabetes history: without (blue) or with (green); (E) BMI: $<21$ (blue) or $\geq 21$ (green); (F) family history: without (blue) or with (green); (G) tumor size: $<3 \mathrm{~cm}$ (blue) or $\geq 3 \mathrm{~cm}$ (green); $(\mathbf{H})$ tumor location: peripheral (blue) or central (green); (I) cT stage: T1 (blue) or T2,3,4 (green); (J) cN stage: N0 (blue) or N1,2 (green); (K) cTNM stage: I stage (blue) or II, III stage (green); (L) tumor pathological type: adenocarcinoma (blue) or squamous cell carcinoma (green).
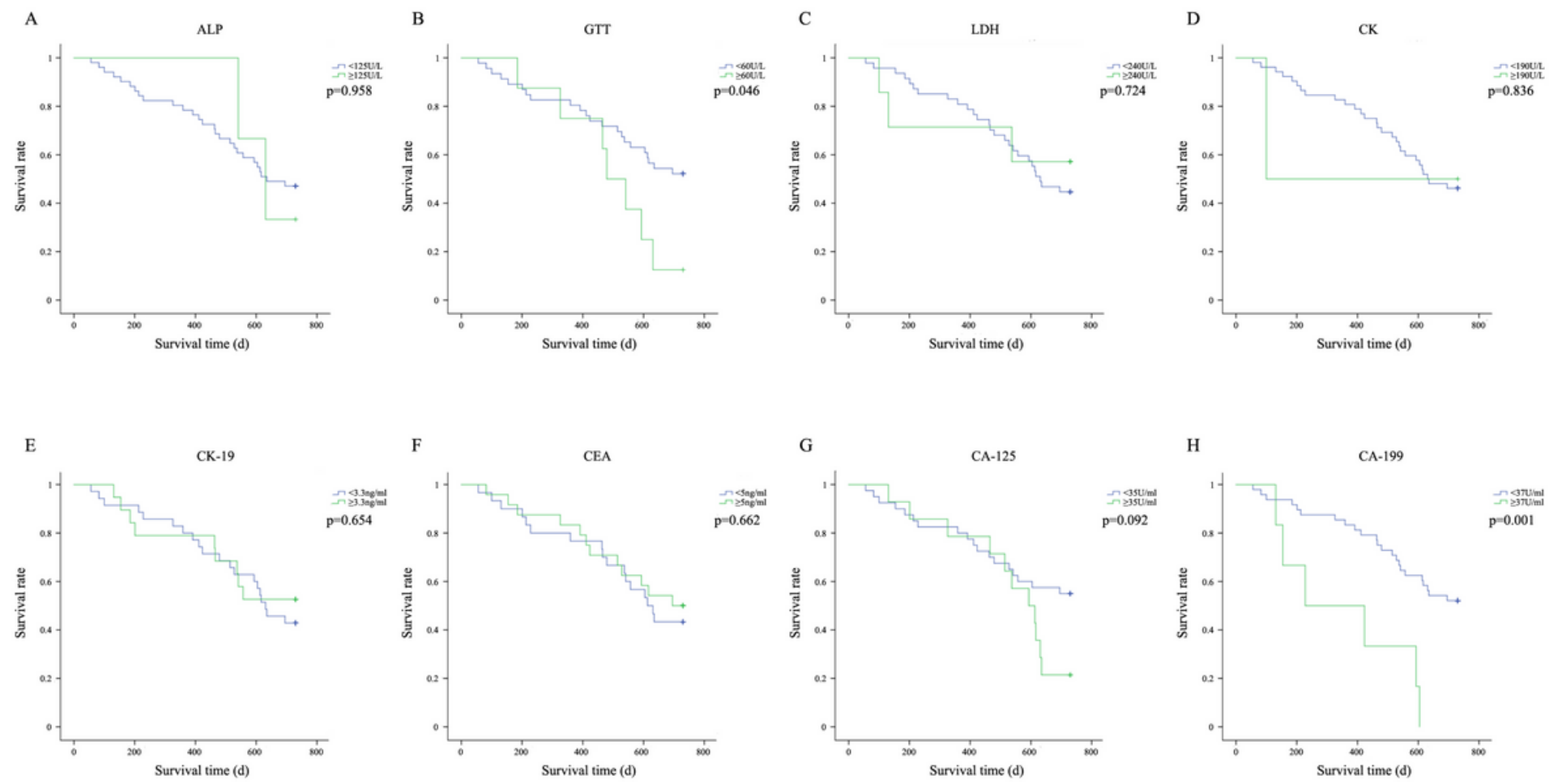

\section{Figure 2}

Kaplan-Meier survival curve of serum factors for 54 NSCLC patients with unexpected pleural dissemination during thoracoscopic surgery. (A) ALP: $<125 \mathrm{U} / \mathrm{L}$ (blue) or $\geq 125 \mathrm{U} / \mathrm{L}$ (green); (B) GTT: $<60 \mathrm{U} / \mathrm{L}$ (blue) or $\geq 60 \mathrm{U} / \mathrm{L}$ (green); (C) LDH: $<240 \mathrm{U} / \mathrm{L}$ (blue) or $\geq 240 \mathrm{U} / \mathrm{L}$ (green); (D) CK: $<190 \mathrm{U} / \mathrm{L}$ (blue) or $\geq 190 \mathrm{U} / \mathrm{L}$ (green); (E) CK-19: $<3.3 \mathrm{ng} / \mathrm{ml}$ (blue) or $\geq 3.3 \mathrm{ng} / \mathrm{ml}$ (green); (F) CEA: $<5 \mathrm{ng} / \mathrm{ml}$ (blue) or $\geq 5 \mathrm{ng} / \mathrm{ml}$ (green); (G) CA-125: $<35 \mathrm{U} / \mathrm{ml}$ (blue) or $\geq 35 \mathrm{U} / \mathrm{ml}$ (green); (H) ALP: $<35 \mathrm{U} / \mathrm{ml}$ (blue) or $\geq 35 \mathrm{U} / \mathrm{ml}$ (green). 
A

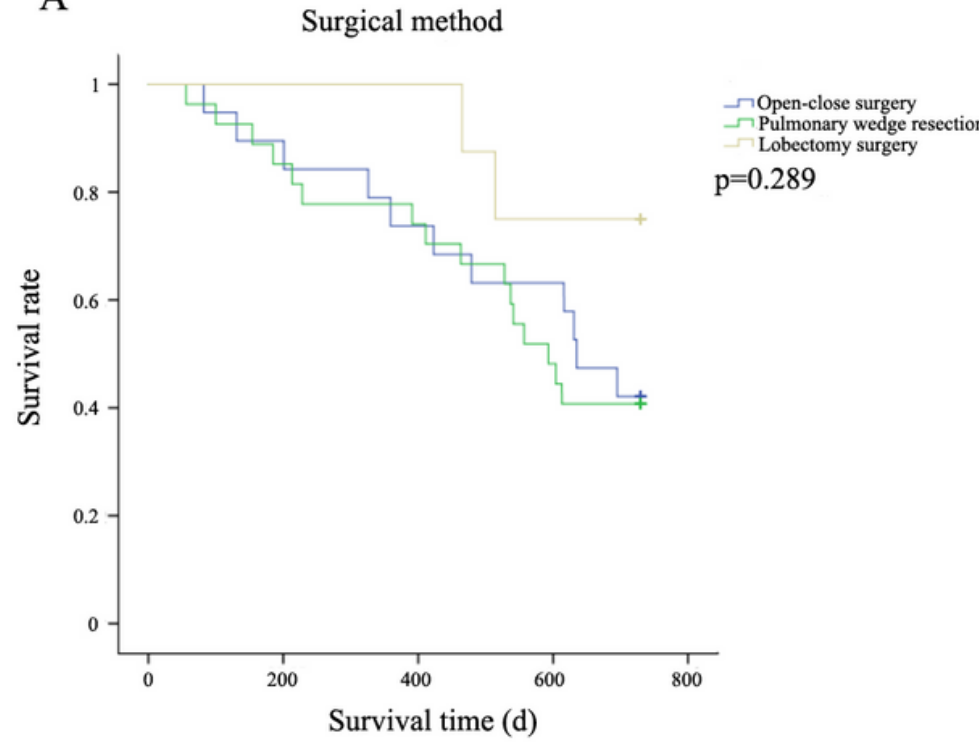

B

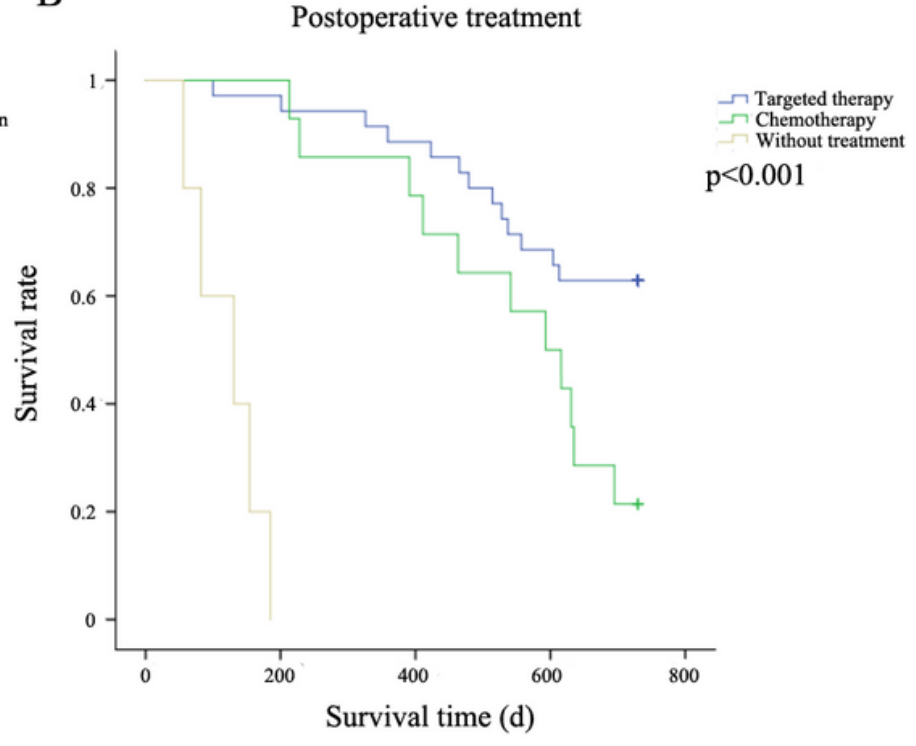

\section{Figure 3}

Kaplan-Meier survival curve of treatment for 54 NSCLC patients with unexpected pleural dissemination during thoracoscopic surgery. (A) surgical method: open-close surgery (blue), pulmonary wedge resection (green) or lobectomy (yellow); (B) postoperative treatment: targeted therapy (blue), chemotherapy (green) or without treatment (yellow). 